Revue internationale P.M.E.

Économie et gestion de la petite et moyenne entreprise

\title{
Stratégies réticulaires et vulnérabilité de la petite entreprise : une illustration
}

\section{Gilles Paché}

Volume 9, numéro 1, 1996

URI : https://id.erudit.org/iderudit/1008251ar

DOI : https://doi.org/10.7202/1008251ar

Aller au sommaire du numéro

Éditeur(s)

Presses de l'Université du Québec

ISSN

0776-5436 (imprimé)

1918-9699 (numérique)

Découvrir la revue

Citer cet article

Paché, G. (1996). Stratégies réticulaires et vulnérabilité de la petite entreprise : une illustration. Revue internationale P.M.E., 9(1), 7-20.

https://doi.org/10.7202/1008251ar
Résumé de l'article

L'objet de l'article est de s'interroger sur la façon dont les petites entreprises s'intégrent aujourd'hui dans les réseaux de compétences. En effet, on entend habituellement dire que les stratégies de réseau s’appuient sur la qualification de tous les partenaires concernés par un même projet productif. L'exemple de la petite entreprise de transport, exerçant ses activités dans un environnement très déstabilisant, apporte un démenti formel à cette position. 


\title{
GREPME \\ Université du Québec à Trois-Rivières \\ Stratégies réticulaires et vulnérabilité de la petite entreprise: une illustration
}

Gilles PACHÉ

Université de la Méditerranée

MOTS CLÉS

\section{Dépendance - Logistique - Partenariat-Petite entreprise Réseau-Transport routier-Vulnérabilité}

\begin{abstract}
RÉSUMÉ
L'objet de l'article est de s'interroger sur la façon dont les petites entreprises s'intègrent aujourd'hui dans les réseaux de compétences. En effet, on entend habituellement dire que les stratégies de réseau s'appuient sur la qualification de tous les partenaires concernés par un même projet productif. L'exemple de la petite entreprise de transport, exerçant ses activités dans un environnement très déstabilisant, apporte un démenti formel à cette position.
\end{abstract}

\section{ABSTRACT}

The aim of this article is to ponder over the way small firms get integrated to competencies networks. Indeed, it is usually heard that network strategies lean

\section{L'AUTEUR}

Gilles Paché est maître de conférences et directeur de recherches en sciences de gestion à l'Université de la Méditerranée (Aix-Marseille II). II s'intéresse plus particulièrement aux stratégies logistiques et mercatiques dans l'industrie et la distribution alimentaire. Sur ces thèmes, il a publié un grand nombre d'articles scientifiques ainsi que quatre ouvrages. Adresse : CRET-LOG, 413, avenue Gaston-Berger, 13625 Aix-enProvence, Cedex 1, France. 
on the qualification of every partner involved in the same productive project. The example of small transport firm, set in an environment creating a high level of vulnerability, denies this position formally.

\section{RESUMEN}

El objetivo del artículo es el de pregoniarse a cerca de la manera en que las pequeñas empresas se integran hoy en las redes de competencias. En efecto, se suele oir decir que las estrategias de red se basan en la cualificación de todos los socios implicados en un mismo proyecto productivo. El ejemplo de la pequeña empresa de transporte, situada en un entorno muy dado a la vulnerabilidad, aporta un mentis mordaz a esta posición. 


\section{Introduction}

À l'heure où les acteurs économiques sont de plus en plus interdépendants, l'étude des rapports interfirmes revêt un intérêt évident pour les chercheurs en gestion et en analyse industrielle. Elle permet de repérer l'émergence de nouvelles trajectoires stratégiques qui remettent largement en question un certain nombre de schémas hérités du passé.

Ces quinze dernières années ont ainsi vu se multiplier, tant en Europe que sur le continent nord-américain, une multitude de recherches sur le thème fédérateur de la «mise en réseau» (networking) des firmes manufacturières. Il est vrai que les formes organisationnelles hybrides, empruntant au marché et à la hiérarchie quelques-uns de leurs traits caractéristiques, recourent à des mécanismes de coordination originaux dont on ne sait encore presque rien.

Beaucoup de publications sur les stratégies réticulaires se consacrent à l'atmosphère de l'échange, en faisant notamment référence aux deux dyades pouvoir/dépendance et coopération / conflit (Sandström, 1992). Il n'y a aucun hasard à cela, car la réussite du «projet productif», qui associe durablement des partenaires aux compétences complémentaires, est en grande partie liée au climat dans lequel baignent les transactions. Par contre, peu de choses sont dites et écrites sur la position des petites entreprises face aux réseaux : peuvent-elles (doivent-elles) s'y insérer à tout prix ? Si non, existe-t-il d'autres politiques relationnelles permettant d'assurer leur pérennité ?

C'est à ce type de questions que l'article souhaite fournir, non pas des réponses tranchées, mais bien des pistes de réflexion. Il repose sur l'hypothèse centrale que, sous certaines conditions, les stratégies réticulaires accroissent le degré de vulnérabilité des petites entreprises (notées PE dans la suite du texte), allant même jusqu'à les mettre en péril à court terme.

Le fil conducteur sera constitué par l'étude de l'une des composantes des réseaux : les entreprises qui se chargent de sa logistique (au sens de pilotage des flux). En effet, ces entreprises prestataires de services-du moins en France - ont pris l'habitude de faire sous-traiter les opérations d'acheminement des marchandises par des artisans-transporteurs totalement captifs et précarisés. Selon nous, il s'agit là d'une illustration tout à fait symptomatique des effets pervers parfois induits par les stratégies réticulaires, à savoir l'institutionnalisation de rapports interfirmes «à deux vitesses». Elle menace la survie des $\mathrm{PE}$ de transport, mais peut-être aussi celle des réseaux eux-mêmes.

\section{Les prestataires logistiques au cœur des réseaux}

Les politiques de «mise en réseau » connaissent un engouement incontestable dans les économies occidentales. Leur principal attrait réside dans le fait que 
les relations d'échange qui en découlent s'appuient sur des procédures incitatives (plutôt que coercitives) dont l'objet est de mieux coordonner les activités et les ressources des diverses composantes.

\subsection{Analyse sommaire des stratégies réticulaires}

Tout se passe aujourd'hui comme si de multiples firmes manufacturières, plutôt que de persévérer dans une croissance de nature patrimoniale, préféraient se concentrer sur quelques activités perçues comme essentielles tandis que les autres activités de leur chaîne de valeur sont externalisées selon des normes et des formats rigoureusement définis (Paché et Paraponaris, 1993). Plutôt que de posséder un ensemble d'outils de production ou de distribution physique, ces firmes-que l'on qualifiera de «recentrées »-se donnent les moyens de les mobiliser auprès de partenaires soigneusement sélectionnés, créant ainsi de véritables réseaux de compétences.

Les transactions qui en résultent n'obéissent cependant qu'en partie aux signaux du marché, soit les prix, puisque sont intentionnellement mis en œuvre des mécanismes partenariaux de stabilisation des relations d'échange:

- d'un côté, les engagements contractés obéissent à un principe de récursivité puisque leur durée est souvent de plusieurs années;

- de l'autre, les rapports interfirmes font référence à des règles complexes de continuité et d'interpénétration des systèmes de gestion du client et du fournisseur (Asanuma, 1989).

C'est sans doute à l'école suédoise du marketing industriel que l'on doit d'avoir le plus clairement précisé les tenants et les aboutissants des stratégies réticulaires. S'appuyant à l'origine sur l'étude des relations entre acheteurs et vendeurs de biens intermédiaires, différents chercheurs de cette école en sont venus à conceptualiser un modèle interactionniste dans lequel les activités et les ressources sont coordonnées en vue de mutualiser des risques, mais aussi des occasions de développement. Dans un article de synthèse, Håkansson et Snehota (1990) concluent que :

- tout acteur, bien que poursuivant ses propres buts, exerce son activité dans un environnement qui conditionne plus ou moins fortement sa conduite («no business is an island», pour reprendre l'expression des auteurs);

- les divers acteurs sont en contact étroit, ce qui leur permet d'accéder aux ressources des uns et des autres, les liens interorganisationnels constituant l'épine dorsale des échanges ; 
- les compétences distinctives de chacun des acteurs sont valorisées par le biais des relations d'échange entretenues avec les autres membres de la structure;

- les comportements des acteurs s'inscrivant dans une logique congruente, la performance est d'abord celle du réseau dans sa totalité, plutôt que de l'un ou l'autre d'entre eux.

Le modèle du réseau fait ressortir une radicale évolution par rapport à des pratiques séculaires, mais encore très vivaces, comme la mise en concurrence systématique des fournisseurs lors d'appels d'offres. Ce qui compte désormais, c'est la coordination optimale de ressources et de capacités préalablement agencées pour faire face aux variations de l'environnement.

Autrement dit, il n'est plus question de s'appuyer sur le modèle standard de la compétition qui gouverne les relations strictement marchandes et pour lequel le partenaire commercial est avant toute chose un adversaire (Johnston et Lawrence, 1988). Au contraire, l'efficacité organisationnelle n'aura de sens que par rapport à un projet productif qui associe symbiotiquement des acteurs complémentaires le long d'une chaîne de valeur. L'exemple de la prestation logistique en donne une parfaite illustration.

\subsection{Vers un partenariat logistique}

À l'image des États-Unis et d'autres pays européens, la France a vu émerger dans les années 1980 une nouvelle génération de transporteurs routiers qui, pardelà la simple activité de traction (c'est-à-dire d'acheminement des marchandises), proposent à leur clientèle un assortiment diversifié mais cohérent de prestations logistiques. L'élargissement significatif de l'offre est d'ailleurs à l'origine de leur dénomination maintenant communément admise: les prestataires logistiques (notés PL dans la suite du texte). Certes, des zones géographiques comme le Québec paraissent pour l'heure en retrait. Plusieurs recherches empiriques soulignent toutefois l'existence de stratégies convergentes permettant d'envisager une généralisation du phénomène (Bigras, 1993).

Pour ceux qui étudient le secteur des transports, il paraît indiscutable que l'avènement progressif des PL est intimement lié au processus de mise en réseau des firmes manufacturières. Très tôt, en effet, des fabricants ont choisi de déléguer leurs activités de transport, de manutention et de stockage à des entreprises spécialisées pour mieux se centrer sur leur métier ou leur mission originelle. Le résultat d'un tel «recentrage» est désormais fort connu. Il a donné la possibilité à de puissants opérateurs de se positionner à l'interface des expéditeurs et des destinataires des marchandises, en aval et en amont des chaînes de valeur. 
Ainsi, ce sont des PL qui, de plus en plus fréquemment, assurent la gestion des magasins avancés dans la filière automobile. Placés au cœur des réseaux de compétences, entre les fournisseurs et les équipementiers d'un côté, et les constructeurs de l'autre, ils organisent les tournées de collecte journalières, le transport d'approche et la tenue du stock tampon de telle sorte que l'unité d'assemblage fonctionne en flux tendus. Mieux encore, certains magasins avancés voient s'exercer en leur sein des activités de préparation et de prépositionnement telles que le montage de réservoirs, de pare-chocs ou de pédaliers (Paché, 1994).

Bref, le PL se présente comme un authentique professionnel de la gestion des flux de marchandises et des informations associées :

- d'une part, il joue sur des effets de massification des flux pour réaliser des économies d'échelle et de champ largement supérieures à ce que pourraient obtenir les membres d'un réseau indépendamment les uns des autres ;

- d'autre part, il n'hésite pas à recourir aux plus récentes technologies de l'information lui permettant de se connecter en temps réel avec ses clients, ce qui facilite la coordination des actifs et accroît l'efficacité des transactions externes (Malone et al., 1987).

Voilà pourquoi, à notre sens, le recours à une «logistique contractuelle », qui repose sur le double principe de récursivité et d'interpénétration des systèmes de gestion, s'est accentué ces dernières années. Disposant de puissantes capacités d'expertise, introduisant des innovations technologiques et maîtrisant de mieux en mieux une démarche prospective qui leur permet d'appréhender les attentes des expéditeurs et des destinataires de marchandises, les PL se donnent les moyens de formuler des solutions originales en matière de gestion des opérations.

Or, c'est justement cette accumulation de compétences qui incite à l'heure actuelle certains PL à s'orienter vers l'acquisition exclusive d'actifs hors traction (Sheffi, 1990). Se muant en organisateurs de chaînes transport, ils préfèrent utiliser leur savoir-faire organisationnel pour concevoir de nouvelles prestations enrichies, quitte à recourir aux actifs traction d'agents extérieurs pour les séquences intermédiaires d'acheminement.

\subsection{Chaîne de valeur et logique de sous-traitance}

Pour Porter (1985), toute chaîne de valeur est composée à la fois d'activités principales et d'activités de soutien dont la mission est de concevoir, fabriquer, commercialiser, distribuer et soutenir un portefeuille de produits. Il existe cinq grandes catégories d'activités principales, dont deux sont de nature logistique. 
1. L'une concerne les activités en amont de la production : programmation et exécution des transports, réception des matières et composants, manutention, entreposage et gestion des stocks. Il s'agit de la logistique interne.

2. L'autre concerne les activités en aval de la production: programmation et exécution des opérations de distribution physique (sortie de stock, manutention, transports d'approche et terminaux). Il s'agit de la logistique externe.

Comme on l'a dit plus haut, ces deux facettes de la logistique sont prises en charge de façon croissante par les PL. La question est évidemment de savoir si elles présentent des caractéristiques homogènes en matière de rentabilité. La réponse est négative. Compte tenu du jeu concurrentiel et des savoir-faire nécessaires pour les gérer, l'attractivité des différents maillons apparaît largement différenciée, forte pour certains, médiocre pour d'autres.

Les PL sont conscients de cet état de fait, tant il est vrai qu'il suffit habituellement d'un audit rapide pour en vérifier la justesse. Leur stratégie consistera alors à voir si le marché ne propose pas une solution de remplacement satisfaisante pour les maillons les moins lucratifs. À l'inverse, l'internalisation s'exercera au détriment des opérations les plus valorisantes, c'est-à-dire celles qui permettent à la fois de formuler une politique de différenciation de l'offre de services et de bénéficier d'un taux élevé de rendement de l'investissement.

Ce cadre d'analyse semble judicieux pour expliquer les pratiques de sous-traitance en cascades que conduisent les PL vis-à-vis d'artisanstransporteurs. En effet, la traction ne réclame aucune compétence particulière; elle est donc accessible aisément au plus grand nombre (voir ci-dessous). En outre, l'absence de coûts irrécupérables facilite la substitution entre tractionnaires, d'autant plus qu'il existe une surcapacité structurelle de l'offre par rapport à la demande (la quantité de fret à transporter).

Dans de telles conditions, on comprend sans peine que les PL aient intérêt à constituer des «nébuleuses » d'affrétés spécialisés par ligne, par aire géographique ou par type de produit, afin de se consacrer aux opérations hors traction. En effet, grâce à la mise en concurrence des PE de transport, ils bénéficient d'une offre pléthorique au moindre coût sans que cela nuise à leur propre rentabilité, déjà assurée par la diversification vers les services logistiques. Signe des temps, nul ne s'étonne plus d'apprendre que tel PL recourt régulièrement à une centaine d'artisans-transporteurs pour l'ensemble ou une partie des acheminements de marchandises, dans des conditions d'extrême précarité le plus souvent. 
Si nous avions à résumer la situation, nous dirions que par l'exercice d'effets de dépendance sur les PE de transport, les PL optent pour une stratégie de domination par les coûts. Paradoxalement, ces mêmes PL annoncent leur souhait de pérenniser une appartenance à des réseaux de compétences en jouant sur la qualité et la singularité des prestations. Il y a donc, en reprenant l'analyse de Marchesnay (1991), articulation de deux bases de compétitivité antinomiques :

- une base de compétitivité fondée sur la qualité de service dans la relation entre le chargeur et le PL;

- une base de compétitivité fondée sur des coûts inférieurs dans la relation entre le PL et les PE de transport.

Selon nous, un tel paradoxe remet largement en question la logique de «qualité totale » explicitement prônée par ceux qui, les premiers, ont introduit les structures réticulaires -c'est-à-dire la qualification de toutes les parties prenantes d'un même projet productif-puisque les conditions tarifaires imposées par le PL conduisent dans les faits à une survie temporaire et à un renouvellement rapide des affrétés. L'issue pourrait en être une fragilisation extrême des chaînes transport et, en définitive, des réseaux de compétences... à moins que les $\mathrm{PE}$ soient capables, dans un sursaut salutaire, d'adopter une démarche proactive d'amélioration de leur prestation.

\section{Les PE de transport: un avenir incertain}

La PE de transport semble enfermée dans une relation de totale dépendance vis-à-vis de son (ou de ses) donneurs d'ordres au regard de la substituabilité et de «l'essentialité » des flux. Qui plus est, l'environnement dans lequel elle évolue la rend très vulnérable. Malgré cela, il n'est pas sûr que son dirigeant accepte l'idée d'une politique associative favorisant l'accès à un espace de transactions indépendant de celui forgé par les PL.

\section{1. Évaluer les causes externes de vulnérabilité}

L'application de grilles d'analyse tirées du management stratégique à la PE de transport est délicate, tout comme pour la PE, en général, du reste. On sait que le propriétaire-dirigeant de ce type d'organisation privilégie un processus de décision réactif s'appuyant sur l'intuition plus que sur la rationalité (Julien et Morel, 1986). Cela est sans doute encore plus exact pour le secteur des transports routiers, car le chef d'entreprise semble moins attiré par les dimensions stratégiques et mercatiques de son affaire que par les dimensions opérationnelles, l'achat de véhicules, par exemple. 
Divers travaux ont bien essayé de dresser une typologie des politiques relationnelles applicables au cas des PME, notamment en s'appuyant sur le triptyque affrontement, évitement et coopération proposé par Koenig (1990). Ce genre de recherches présente toutefois deux grandes limites:

- rien ne dit que chacune des options stratégiques retenues soit adaptée à l'ensemble hétérogène des PME (il y a un monde entre la petite et la moyenne entreprise !);

- l'environnement est beaucoup trop négligé alors même qu'il est plus ou moins propre à accroître la vulnérabilité de la PE selon son secteur d'appartenance.

Le second point mérite une attention toute particulière dans la mesure où l'environnement va, en définitive, conditionner la faisabilité des politiques relationnelles. Si l'on suit Marchesnay (1994), le secteur d'appartenance induit, pour une entreprise donnée, une vulnérabilité plus ou moins grande. Cette vulnérabilité se mesure par trois critères basiques : le degré de complexité (nombre d'acteurs, de variables et de relations), le degré de turbulence (fréquence et amplitude des perturbations techniques et mercatiques) et le degré d'accessibilité (présence ou non de barrières à l'entrée).

À partir de là, il devient possible de repérer deux cas de figure extrêmes, entre lesquels se positionnent une multiplicité de situations plus contrastées.

1. Certaines PE se retrouvent dans un environnement concurrentiel peu complexe, peu accessible et peu turbulent. Il a une faible propension à les rendre vulnérables.

2. À l'inverse, d'autres PE subissent un environnement très complexe, très accessible et très turbulent. Il a une forte propension à les rendre vulnérables.

Qu'en est-il précisément pour la PE de transport, compte tenu des informations présentées jusqu'ici ? De façon incontestable, le degré de complexité dans les techniques logistiques tend à s'accroître avec le processus de recentrage des firmes manufacturières, d'où le succès que connaissent les PL. Parallèlement, tout au moins pour ce qui concerne l'activité de traction dans laquelle se cantonnent les opérateurs de petite taille, le degré d'accessibilité est élevé, vu la faiblesse des barrières techniques, économiques et institutionnelles à l'entrée. Enfin, le degré de turbulence s'intensifie depuis quelques années avec la segmentation des besoins de la clientèle, conduisant d'ailleurs nombre de transporteurs routiers généralistes à se spécialiser dans quelques familles de produits. 
En d'autres termes, les PE de transport se situent sans contestation possible dans un environnement fortement créateur de vulnérabilité. Il est à craindre que cela se traduise par une accélération du processus d'éviction au profit de mégatransporteurs, pour la plupart PL. C'est la conclusion à laquelle arrive Netzer (1994) à partir d'un modèle de simulation à deux scénarios, l'un avec nouveaux entrants et l'autre sans. Dans les deux cas, la concentration du secteur-mesurée par l'indice d'Herfindhal - tend à augmenter de manière notable.

Pour essayer d'échapper à ce processus, certaines PE de transport s'efforcent de concrétiser une politique relationnelle de croissance par coopération, les stratégies de différenciation ne pouvant être envisagées faute de ressources propres suffisantes. Les résultats obtenus semblent moyens, sûrement parce qu'aux causes externes de vulnérabilité viennent s'adjoindrent des causes internes telles que l'inefficience du système de décision (Capiez, 1992).

\subsection{L'échec relatif des coopérations horizontales}

Il serait illusoire d'entretenir de trop grands espoirs quant aux aptitudes des propriétaires-dirigeants de $\mathrm{PE}$ de transport à développer une réflexion prospective relativement structurée. Les choses ne devraient d'ailleurs pas s'améliorer à court terme dans la mesure où la majorité des nouveaux créateurs sont d'anciens chauffeurs, sans aucune formation aux fonctions de direction (Braibant, 1991). Il est vrai que même les grandes entreprises du secteur n'ont que récemment adopté une démarche marketing, préférant pendant longtemps se consacrer à la résolution prioritaire des problèmes d'exploitation (optimisation des taux de remplissage, gestion de la flotte, etc.).

Heureusement, sous l'impulsion des pouvoirs publics et de quelques syndicats professionnels, les PE prennent petit à petit conscience de l'urgence d'une action volontariste pour sortir de l'impasse concurrentielle dans laquelle elles se trouvent. Il s'agirait notamment de susciter une sorte de partenariat vertical avec un ou plusieurs PL pour accéder à de nouveaux marchés et à des ressources matérielles et immatérielles faisant cruellement défaut. Les logiques d'action à l'œuvre semblent exclure une telle option.

1. Travailler pour des PL ne permet en rien aux PE de transport d'élargir leur périmètre commercial. Ce sont les prestataires de services logistiques qui maîtrisent l'ensemble du processus de servuction en disposant à leur guise des capacités (et de la flexibilité) de tractionnaires totalement asservis.

2. En outre, les PL se gardent bien de procurer à leurs affrétés les moyens de sortir de leur isolement (capitaux, soutien technique, informations 
sur les marchandises à acheminer, etc.). Plus la fragilité de la PE de transport est grande, plus le pouvoir de coercition s'exerce au moindre coût et de manière récurrente.

Comment s'extraire dès lors des relations de dépendance tissées par les PL ? La solution la plus réaliste s'apparente à la concrétisation de coopérations horizontales entre entreprises de même taille, aux potentiels similaires ou complémentaires. Nous nous sommes ainsi fait, dans un précédent article (Paché, 1990), l'apôtre de collaborations techniques qui pourraient déboucher soit sur des systèmes d'échange de fret à l'échelle nationale (puis européenne), soit sur une «percolation de savoir-faire», des PE de transport s'associant par exemple à des entrepositaires pour ébaucher un service complet de distribution physique.

Au demeurant, et de façon symptomatique, c'est bien une position similaire qu'adopte Fourcade (1994) lorsqu'elle souligne l'importance de l'accès à l'information pour les PE voulant maîtriser les technologies les plus avancées. Sachant qu'une démarche individualiste conduit systématiquement à l'échec, la seule solution viable est la formulation de procédures collectives de veille.

Hélas ! il faut en convenir, hormis quelques expériences ponctuelles, ce type de politique relationnelle ne connaît pas de véritable engouement dans le transport, les logiques d'action sectorielles privilégiant l'indépendance et l'autonomie de décision. L'échec le plus retentissant est sans doute celui des coopératives dont on escomptait, dans les années 1980, une rapide expansion en France.

L'égalité des droits et obligations des sociétaires d'une coopérative de transport (comme dans toute coopérative) se traduit en effet par une absence de leadership paralysant rapidement les structures, jusqu'à entraîner leur démantèlement, à moins que les objectifs stratégiques soient fixés par une direction générale constituée de gestionnaires non sociétaires. Dans ce cas, la coopérative de transport risque de dériver doucement vers une répartition asymétrique du pouvoir de décision, ce qui n'est sans doute pas le but de l'exercice!

Les enquêtes que nous avons pu réaliser en France depuis une douzaine d'années auprès de transporteurs routiers invalident ainsi l'existence de plusieurs des profils de propriétaires-dirigeants repérés par Chappoz (1991), en fait, tous ceux qui postulent à des degrés divers une attitude adaptative ou opportuniste valorisant l'interaction de la PE avec son environnement. Nous illustrerons ce point avec l'exemple de la messagerie express, en parfaite harmonie avec les systèmes industriels et commerciaux fonctionnant sur le mode des flux tendus. 
Les opérateurs actuellement présents sur le marché sont d'origines très diverses. Les plus dynamiques proviennent de l'aérien ou recourent à ce mode pour contracter considérablement les délais globaux d'acheminement (on y retrouve les fameux «integrators» tels que Federal Express, UPS, etc.). Quelques transporteurs routiers commencent toutefois à occuper le terrain en collaboration avec les sociétés ferroviaires pour les tractions de longue distance. Il s'agit principalement de grandes entreprises, de stature européenne pour quelques-unes, nationale pour la plupart.

Quant aux PME, un petit noyau s'est organisé en réseau d'échange de fret pour pouvoir mailler le territoire français dans de bonnes conditions (France Rapide, Sernadis, France Étoile, etc.), mais son influence demeure très marginale, sans doute parce qu'il lui est difficile de garantir durablement l'enchaînement coordonné des séquences de traction, de passage sur quai et de recomposition des chargements. À cela, trois raisons essentielles :

- d'une part, chaque acteur est porteur de spécificités culturelles ou salariales, parfois incompatibles avec celles des autres acteurs;

- d'autre part, se pose le problème central de la définition d'une politique tarifaire homogène acceptée par tous ;

- enfin, toute déficience «locale» de l'un des acteurs au regard de la qualité du service ternira rapidement l'image de marque globale du réseau.

Voilà pourquoi les grandes entreprises de transport routier et les «integrators », lesquels contrôlent les nœuds principaux de leur système de circulation (et n'hésitent pas à recourir à des affrétés pour les tractions de longue distance), disposent d'un avantage concurrentiel solide. Voilà également pourquoi les coopérations entre PE ne connaissent qu'une diffusion confidentielle, même si l'on peut espérer que les politiques relationnelles soient, par un apprentissage organisationnel, de mieux en mieux maîtrisées et se présentent comme une solution de rechange séduisante aux pratiques de sous-traitance. Soyons sûr, quoi qu'il advienne, que le changement sera de nature incrémentale plutôt que quantique, comme il est de coutume dans les organisations (Mintzberg, 1994).

\section{Conclusion}

Il est habituel d'entendre dire que, face au développement des exigences logistiques des entreprises en réseau, seule une véritable stratégie de partenariat offre aux PE de réelles garanties d'efficacité. L'idée est séduisante et pourrait même former la trame d'une politique industrielle audacieuse. Les conditions 
objectives de fonctionnement du marché du transport routier français montrent toutefois qu'il s'agit là, pour l'instant, d'un vœu pieux.

Le principal risque est de voir les PE de transport progressivement marginalisées par rapport aux grands flux d'échange, un puissant noyau de PL maîtrisant les interfaces logistiques pour son unique bénéfice. Quand on connaît l'ancrage des artisans-transporteurs dans le tissu local, il est aisé d'en imaginer les conséquences sur certaines aires géographiques en matière d'emploi.

Mais ce n'est pas tout. Le fonctionnement efficace des réseaux exige des acheminements ponctuels et fiables. Or, la politique de mise en concurrence systématique des affrétés pratiquée par les PL paraît largement incompatible avec la philosophie de la «qualité totale». L'activité de traction pourrait ainsi devenir le maillon faible des chaînes transport et mettre en danger la compétitivité des systèmes productifs dans un environnement de plus en plus internationalisé.

\section{Bibliographie}

ASANUMA, B. (1989), « Manufacturer-supplier relationships in Japan and the concept of relation specific skill», Journal of the Japanese and International Economies, vol. 3, $\mathrm{n}^{\circ} 1$, p. 1-30.

BIgRAS, Y. (1993), "Les PME face à l'intégration mondiale des systèmes de transport », Communication à la $1^{\text {re }}$ Conférence internationale francophone de la PME, Carthage, octobre.

BRAIBANT, M: (1991), «Le transport routier de marchandises à la veille de l'échéance européenne », Économie et Statistique, no 239, p. 3-17.

CAPIEZ, A. (1992), «Les chances de succès des petites entreprises : vers un diagnostic d'émergence », Revue Internationale PME, vol. 5, n 2, p. 103-132.

ChAPPOZ, Y. (1991), «La gestion de l'interactivité entreprise/ environnement », Revue Internationale PME, vol. 4, $\mathrm{n}^{\mathrm{0}} 3$, p. 53-75.

FOURCADE, C. (1994), «Localisation versus globalisation: les stratégies résiliaires des petites entreprises », Communication présentée à la $39^{\mathrm{e}}$ ICSB World Conference, Strasbourg, juin.

HÅkANSSON, H. et I. SNEhota (1990), «No business is an island: the network concept of business strategy », Scandinavian Journal of Management, vol. 4, $\mathrm{n}^{0} 3$, p. 187-200.

Johnston, R. et P. LAWRENCE (1988), «Beyond vertical integration - The rise of the value-adding partnership », Harvard Business Review, vol. 66, n 4, p. 94-101. 
JULIEN, P.A. et B. MOREL (1986), La belle entreprise: la revanche des PME en France et au Québec, Montréal, Boréal.

KoENIG, G. (1990), Management stratégique : vision, manœuvres et tactiques, Paris, Nathan.

MALONE, T., J. YATES et R. BENJAMIN (1987), «Electronic markets and electronic hierarchies », Communications of the ACM, vol. 30, nº 6, p. 484-497.

MARCHESNAY, M. (1991), «Stratégie des petites et moyennes entreprises », dans

R. Arena et al. (éd.), Traité d'économie industrielle, $2^{\mathrm{e}}$ édition, Paris, Economica, p. 458-467.

MarcheSnAY, M. (1994), «Le management stratégique », dans P.A. Julien (éd.), Les PME: bilan et perspectives, Cap-Rouge, Québec, Les Presses Inter Universitaires, p. 133-162.

Mintzberg, H. (1994), The Rise and Fall of Strategic Planning, New York, The Free Press.

Netzer, J.L. (1994), « Modèle d'évolution du secteur des transports routiers de marchandises », Communication aux $4^{\mathrm{es}}$ journées du Séminaire d'études et de Statistiques appliquées à la modélisation en économie, Lyon, septembre.

PACHÉ, G. (1990), «Stratégies d'adaptation de la petite entreprise », Revue Française de Gestion, $\mathrm{n}^{\circ} 78$, p. 52-60.

PAChé, G. et C. PARAPONARIS (1993), L'entreprise en réseau, Paris, Presses universitaires de France.

PACHÉ, G. (1994), La logistique : enjeux stratégiques, Paris, Vuibert.

Porter, M. (1985), Competitive Advantage, New York, The Free Press.

SANDSTRÖM, M. (1992), «Atmosphere: the emotional superstructure of business relationships », Communication présentée à la $8^{\mathrm{e}}$ IMP Conference, Lyon, septembre.

SHEFFI, J. (1990), «Third-party logistics : present and future prospects », Journal of Business Logistics, vol. 11, $\mathrm{n}^{\circ} 2$, p. 27-39. 